\title{
Bulky high-mannose-type N-glycan blocks the taste-modifying activity of miraculin.
}

\section{$\operatorname{AUTHOR}(S):$}

Ito, Keisuke; Sugawara, Taishi; Koizumi, Ayako; Nakajima, Ken-Ichiro; Shimizu-Ibuka, Akiko; Shiroishi, Mitsunori; Asada, Hidetsugu; ... Iwata, So; Kobayashi, Takuya; Abe, Keiko

\section{CITATION:}

Ito, Keisuke ... [et al]. Bulky high-mannose-type N-glycan blocks the taste-modifying activity of miraculin.. Biochimica et biophysica acta 2010, 1800 (9): 986-992

\section{ISSUE DATE:}

2010-09

URL:

http://hdl.handle.net/2433/128855

\section{RIGHT:}

(c) 2010 Elsevier B.V.; この論文は出版社版でありません。引用の際には 出版社版をご確認ご利用ください。; This is not the published version. Please cite only the published version. 


\section{Bulky High-mannose-type N-glycan Blocks the Taste-modifying Activity of Miraculin}

$\underline{\text { Keisuke Ito }^{1}}$, Taishi Sugawara ${ }^{1}$, Ayako Koizumi ${ }^{1}$, Ken-ichiro Nakajima $^{1}$, Akiko Shimizu-Ibuka ${ }^{1}$, Mitsunori Shiroishi ${ }^{2}$, Hidetsugu Asada ${ }^{2}$, Takami Yurugi-Kobayashi ${ }^{2}$, Tatsuro Shimamura ${ }^{2}$, Tomiko Asakura $^{1}$, Katsuyoshi Masuda ${ }^{3}$, Masaji Ishiguro ${ }^{3}$, Takumi Misaka ${ }^{1}$, So Iwata ${ }^{2,4,5}$, Takuya Kobayashi $^{2,4 *}$, Keiko Abe ${ }^{1 *}$

1 Department of Applied Biological Chemistry, Graduate School of Agricultural and Life Sciences, The University of Tokyo, Bunkyo-ku, Tokyo 113-8657, Japan.

2 Iwata Human Receptor Crystallography project, ERATO, JST, Konoe-cho, Yoshida, Sakyo-ku, Kyoto 606-8501, Japan.

3 Suntory Institute for Bioorganic Research, Wakayamadai 1-1-1, Shimamoto-cho, Mishima-gun, Osaka 618-8503, Japan

4 Department of Medical Chemistry, Kyoto University Faculty of Medicine, Konoe-cho, Yoshida, Sakyo-ku, Kyoto 606-8501, Japan.

5 Division of Molecular Biosciences, Membrane Protein Crystallography Group, Imperial College London, London SW7 2AZ, UK.

* Corresponding authors

Takuya Kobayashi

address: Laboratory of Cell Biology, 3rd Flr., Bldg. A, Graduate School of Medicine, Kyoto University, Yoshida-konoe-cho, Sakyo-ku, Kyoto, 606-8501, Japan

E-mail: t-coba@mfour.med.kyoto-u.ac.jp

Tel: +81-75-753-4386

Fax: $+81-75-753-4660$

Keiko Abe

address: Laboratory of Biological Function, 3rd Flr., Bldg. 7B, Graduate School of Agricultural and Life Sciences, The University of Tokyo 1-1-1, Yayoi, Bunkyo-ku, Tokyo, 113-8657, Japan E-mail: aka7308@mail.ecc.u-tokyo.ac.jp

Tel: +81-3-5841-5129

Fax: +81-3-5841-8006 


\section{Background}

Miraculin (MCL) is a taste-modifying protein that converts sourness into sweetness. The molecular mechanism underlying the taste-modifying action of MCL is unknown.

\section{Methods}

Here, a yeast expression system for MCL was constructed to accelerate analysis of its structure-function relationships. The Saccharomyces cerevisiae expression system has advantages as a high-throughput analysis system, but compared to other hosts it is characterized by a relatively low level of recombinant protein expression. To alleviate this weakness, in this study we optimized the codon usage and signal-sequence as the first step. Recombinant MCL (rMCL) was expressed and purified, and the sensory taste was analyzed.

\section{Results}

As a result, a $2 \mathrm{mg} / \mathrm{L}$ yield of $\mathrm{rMCL}$ was successfully obtained. Although sensory taste evaluation showed that rMCL was flat in taste under all the $\mathrm{pH}$ conditions employed, taste-modifying activity similar to that of native MCL was recovered after deglycosylation. Mutagenetic analysis revealed that the N-glycan attached to Asn42 was bulky in rMCL.

\section{Conclusions and general significance}

The high-mannose-type N-glycan attached in yeast blocks the taste-modifying activity of rMCL. The bulky N-glycan attached to Asn42 may cause steric hindrance in the interaction between active residues and the sweet taste receptor hT1R2/hT1R3.

Keywords: miraculin; sweet protein; taste; yeast expression system; N-glycan; steric hindrance Running title: N-glycan blocks the taste-modifying activity 


\section{Introduction}

Miraculin (MCL) is a naturally-occurring protein in the fruit of the West-African plant Richadella dulcifica [1]. It is a homodimer that consists of two glycosylated 191-amino acid polypeptides cross-linked by disulfide bonds $[2,3,4]$. MCL is of particular interest because it has unique taste-modifying properties. Though flat in taste at neutral $\mathrm{pH}$, it shows taste-modifying activity and converts sourness to sweetness at acidic pH [5]. Although this interesting sensory effect has been characterized, the molecular mechanism underlying the taste-modifying action of MCL is unknown.

There have been limited findings on the structure-function relationships of MCL. Our mutagenetic study using the Aspergillus oryzae expression system [6] showed that the histidine residue His30, located at the interface of the two monomeric MCL subunits, is one of the candidate active residues. As well as A. oryzae, other recombinant-MCL (rMCL) expression systems have been reported previously $[7,8]$. To accelerate analysis of the structure-function relationships of the taste-modifying activity, however, it would be necessary to have a high-throughput expression system.

The yeast Saccharomyces cerevisiae expression system has advantages as a high-throughput analysis system [9,10], and we reported an advanced method useful for expressing proteins bearing various mutations [11]. Using this advanced method, mutated proteins can be expressed within five days of mutation design. In addition, yeast can secrete folded proteins like A. oryzae. These characteristics of yeast expression systems render them suitable for high-throughput mutagenetic analysis. S. cerevisiae, however, has the disadvantage of relatively low expression levels of recombinant protein compared to other hosts [12]. To alleviate this weakness, in this study we focused on codon usage and signal-sequence as the first step of the system. These factors play an important role in high-level expression of secretory protein. Finally, through optimization of these factors, a $2 \mathrm{mg} / \mathrm{L}$ yield of $\mathrm{rMCL}$ in media was successfully obtained. As the second step, the yeast rMCL was purified and its taste-modifying activity was analyzed. Although taste-modifying activity was not initially detected in the purified rMCL, it was significantly recovered by deglycosylation treatment. The structural 
effects of glycosylation on the taste-modifying activity are discussed, along with the results of the mutagenetic study and simulation modeling.

\section{Materials and Methods}

\section{1. Codon optimization.}

Codon-optimized genes were designed based on the protein sequence of MCL (GenBank Accession No. BAA07603) according to the codon bias of $S$. cerevisiae [13] (http://www.kazusa.or.jp/codon). The genes were synthesized by Takara (Otsu, Japan). Codon adaptation index (CAI) values were estimated using the JCat program [14].

\section{2. Yeast transformation.}

The gene fragments of signal-sequences (codon-optimized) were synthesized by primer dimerization using various primers (Fig. S1). To obtain the PCR fragments of MCL, gene-specific primers were as follows: 5'-GGTGGTGGTGATTATAAAGATGATGATGATAAAGATTCGGCACCCAATCCGGTTCTT G-3' and 5'-AAATTGACCTTGAAAATATAAATTTTCCCCTCATTAGAAGTATACGGTTTTGTTGAA C-3' for native-codon and 5'-GGTGGTGGTGATTATAAAGATGATGATGATAAAGATTCTGCTCCAAATCCAGTTTTG GACATT-3' and 5'-AAATTGACCTTGAAAATATAAATTTTCCCCCTATTAGAAGTAAACAGTTTTTTGG-3' for codon-optimized MCL. Primers contained a gene-specific region (bold) and a homologous region (italic). PCR fragments, signal-sequence and the SmaI-linearized pRS426_GAL1 vectors were co-transformed into S. cerevisiae strain FGY217 [15]. Transformation was performed as described previously [11].

\section{3. Liquid expression.}

For expression-screening of MCL, transformants were selected and grown in -Ura selection medium $(0.2 \%$ yeast synthetic drop-out medium without Ura, $0.7 \%$ yeast nitrogen base without amino acids and $2 \%$ glucose). After growing at $30^{\circ} \mathrm{C}$ for $24 \mathrm{~h}$, cells were harvested by centrifugation and resuspended in an equal volume of expression medium (1\% casamino 
acids, $1.5 \%$ yeast nitrogen base without amino acids and $2 \%$ galactose, $\mathrm{pH} 4.0$ ) at a final $\mathrm{OD}_{600}$ of 7 and grown at $20^{\circ} \mathrm{C}$ for $24 \mathrm{~h}$. The culture supernatant was collected and analyzed using anti-MCL antibody [6]. The expression yields were estimated by the detection level in comparison with that of native-MCL (nMCL). For over-expression conditions, after growing in -Ura selection medium at $30^{\circ} \mathrm{C}$ for $24 \mathrm{~h}$, cells were transferred into growth medium $(1 \%$ peptone, $0.5 \%$ yeast extract and $2 \%$ glucose) and grown at $30^{\circ} \mathrm{C}$ for $24 \mathrm{~h}$. Cells were harvested by centrifugation, resuspended in a half-volume of expression medium at a final $\mathrm{OD}_{600}$ of 14 , and grown at $20^{\circ} \mathrm{C}$ for $24 \mathrm{~h}$.

\section{4. On-plate detection of secreted MCL.}

The signal-sequence optimization method is illustrated in Fig. 1. Twelve yeast signal-sequences selected according to the report by Sahara et al. [16] and MCL signal-sequence, were synthesized by primer dimerization (Fig. S1). After co-transformation with a mixture of signal-sequences and MCL PCR fragment, cells were distributed on a -Ura expression plate ( $2 \%$ agarose, $0.2 \%$ yeast synthetic drop-out medium without Ura, $0.7 \%$ yeast nitrogen base without amino acids, $0.1 \%$ glucose, $2 \%$ galactose) and covered by a PVDF membrane. Colonies were grown at $30^{\circ} \mathrm{C}$ for $24 \mathrm{~h}$ and $20^{\circ} \mathrm{C}$ for $72 \mathrm{~h}$. The membrane was analyzed by immunodetection using anti-FLAG antibody.

\section{5. Purification and deglycosylation.}

rMCL was purified by hydrophobic, cation-exchange and size-exclusion column chromatographies [6]. Purity and yield of purified rMCL were evaluated by SDS-PAGE. For deglycosylation, endoglycosidase F1 was added to purified rMCL. After incubation at $30^{\circ} \mathrm{C}$ for $12 \mathrm{~h}$, the enzyme was removed by size-exclusion chromatography.

\section{6. Sensory analysis of the taste-modifying activity.}

The taste-modifying activity of MCL was evaluated by a panel of well-trained members. One hundred microliters of MCL solution, $0.1 \mathrm{mg} / \mathrm{mL}$ for $\mathrm{nMCL}$ or $0.5 \mathrm{mg} / \mathrm{mL}$ for $\mathrm{rMCL}$, was applied to the tongue and held for $1 \mathrm{~min}$, and the mouth was then rinsed with water. Then 400 $\mu \mathrm{L}$ of $100 \mathrm{mM}$ sodium citrate buffer at $\mathrm{pH} 7.0,5.0$ or 3.0 was applied to the tongue and the sweetness was evaluated by comparing the taste of these solutions with those of standard aspartame solutions. Sweetness scores were represented according to the concentration of aspartame: score 9, >2 mM; score 8, $2 \mathrm{mM}$; score 7, 1-2 mM; score 6, $1 \mathrm{mM}$; score 5, 0.5-1 
$\mathrm{mM}$; score 4, $0.5 \mathrm{mM}$; score 3, 0.25-0.5 mM; score 2, $0.25 \mathrm{mM}$; and score 1, $<0.25 \mathrm{mM}$.

\section{Results}

\section{1. Enhanced expression of miraculin through codon optimization}

To improve the expression level, MCL codon usage was optimized for yeast codon frequencies (see MATERIALS AND METHODS). Fig. 2 shows the gene sequence of native (a) and optimized (b) MCL. rMCL was detected as a broad band at $180 \mathrm{kDa}$ by Western blotting (Fig. 2c). By optimizaton of codon usage, the expression level was increased from 0.3 $\mathrm{mg} / \mathrm{L}$-culture up to $0.8 \mathrm{mg} / \mathrm{L}$-culture, with an increase of CAI from 0.068 up to 0.53 (Fig. 2d).

\section{2. Screening of signal-sequence to improve the secretion level}

To improve the expression level of rMCL, the secretion signal-sequence was optimized. Approximately $2 \times 10^{2}$ colonies per experiment were obtained by co-transformation with two gene fragments; signal sequence and MCL fragment (Fig. 3a). Most colonies were found to be rMCL-expressing clones, when analyzed by on-plate immunodetection using anti-FLAG antibody. Twelve strongly-positive clones were selected from the plate and the adapted signal-sequence of these clones was confirmed by sequencing (Fig. 3b). All 12 clones had a relatively strong secretion signal-sequence when compared to the MCL signal-sequence. The optimized signal-sequence was that of SSP120, which is a protein of unknown function [17]. Under screening conditions, the use of SSP120 signal-sequence increased the expression level from $0.8 \mathrm{mg} / \mathrm{L}$-culture up to $1.3 \mathrm{mg} / \mathrm{L}$-culture.

\section{3. Purification, deglycosylation, and sensory analysis of $r M C L$}

In over-expression conditions, a $2 \mathrm{mg} / \mathrm{L}$-culture yield of $\mathrm{rMCL}$ was secreted into the media, and a $0.5 \mathrm{mg} / \mathrm{L}$-culture yield of purified $\mathrm{rMCL}$ was obtained. The rMCL had a very large molecular size of approximately $180 \mathrm{kD}$ even under reducing conditions (Fig. 4a), which, following deglycosylation using endoglycosidase F1 [18], was decreased to $44 \mathrm{kDa}$ and $22 \mathrm{kDa}$ under non-reducing and reducing conditions, respectively. Although sensory taste assessment showed that purified rMCL was flat in taste under all the $\mathrm{pH}$ conditions employed (Fig. 4b), taste-modifying activity similar to that of nMCL was recovered after deglycosylation. 


\section{4. Mutagenetic analysis of glycosylation sites}

To analyze sugar-chain modification of yeast-expressed rMCL, we compared three mutated proteins, N42Q, N186Q, and N42-186Q (C47-92S) by gel-shift analysis (Fig. 5). In the case of the N42-186Q double-mutant, a quadruple-mutant with a high-expressing mutation, C47-92S (paper in preparation), was analyzed because the N42-186Q mutant was not secreted. The band of the N42Q mutant was narrow at $50 \mathrm{kDa}$ and the molecular size slightly increased to reach that of the N42-186Q (C47-92S) non-glycosylated mutant (44 kDa). In contrast, the major band of the N186Q mutant was broad at $180 \mathrm{kDa}$, which was similar to the molecular size of wild-type MCL.

\section{Discussion}

\section{1. Improvement of MCL expression level in the yeast expression system}

Codon usage is an important factor for protein expression level in heterologous expression systems $[19,20]$. S. cerevisiae has a codon bias on tRNAs coding arginine and glycine especially [21], and the expression level of rMCL was improved by codon optimization (Fig. 2d). MCL is a basic protein composed of 191 amino acids with 11 arginine and 17 glycine residues. Optimization of the codons for these residues may make a major contribution to the observed enhancement of expression level and CAI.

Most secretory proteins are initially synthesized in the cytoplasm as precursors containing signal-sequence extensions at their $\mathrm{N}$-terminals, and are transported by signal-sequence recognition proteins to the endoplasmic reticulum (ER). The ER has a stringent quality control system that ensures the correct folding of proteins to be exported via the secretory pathway. Therefore, the signal-sequence plays an important role in improving the secretion level. The signal-sequence also plays a role in heterologous expression systems. In fact, rMCL was secreted in yeast using the MCL signal-sequence itself (Fig. 2c). Sahara et al. [16] analyzed the luciferase secretion efficacy in yeast for 400 signal-sequences extracted from the yeast genome database. Although signal-sequence optimization is effective in improving the secretion level of recombinant protein, the screening is a time-consuming process. A rapid optimization system for the signal-sequence would be necessary to efficiently promote protein secretion.

Here, we developed a signal-sequence optimization system by co-transformation with two gene fragments, based on our mutagenesis method [11]. In this system, a specific gene coding a 
$3 \times$ glycine and FLAG-tag (GGGDYKDDDDK) is used for the homologous site (see

MATERIALS AND METHODS and Figs. 1, S1), where the three glycine residues may promote signal-sequence processing and the FLAG-tag is available for specific on-plate detection. The library consisting of various signal-sequences can be constructed in one step in just a week. On-plate detection is by a high-throughput screening method that can analyze a number of clones simultaneously [22]. This combination of procedures enables us to obtain the ideal signal-sequence for effective secretion of the recombinant protein in just a week. Moreover, signal-sequence fragments can useful for other target proteins and one gene-specific primer coding a $3 \times$ glycine and FLAG-tag is required for a new run of screening. Through the optimization of codon usage and signal-sequence, under screening conditions the expression level of rMCL was increased from $0.3 \mathrm{mg} / \mathrm{L}$-culture up to $1.3 \mathrm{mg} / \mathrm{L}$-culture.

\section{2. The role of $\mathrm{N}$-glycan in the taste-modifying activity of $M C L$}

Many proteins in $S$. cerevisiae are modified by the attachment of N-glycan to asparagine. In particular, some secretory proteins are in a hyper-mannosylated form and may contain up to 200 mannose units [23]. rMCL was secreted in the hyper-mannosylated form in the yeast expression system (Fig. 2c), while the N-glycan of nMCL is of a complex type [3]. Enzymatic deglycosylation of nMCL is difficult without any loss of the taste-modifying activity [1], and it is inhibited by steric hindrance of the 3-D structure, because deglycosylation of denatured MCL proceeded uneventfully (data not shown). Endoglycosidase F1 removes high-mannose-type $\mathrm{N}$-glycan from protein with less sensitivity to the protein conformation [18]. Yeast-expressed rMCL was successfully deglycosylated by this enzyme (Fig. 4a). While purified rMCL did not have any taste-modifying activity, deglycosylated rMCL (dg-rMCL) showed similar activity to nMCL (Fig. 4b). This result suggests that the attached high-mannose-type N-glycan in yeast blocks the taste-modifying activity of rMCL.

The taste-modifying activity of MCL is known to be mediated by the sweet taste receptor, hT1R2/hT1R3, because lactisole suppressed this activity [6]. However, the details of the interactions have not been fully elucidated. The rMCL expressed by A. oryzae has a sensory-active form [6]. This indicates that the high-mannose-type N-glycan itself does not inhibit the taste-modifying activity. As reported previously [7], rMCL expressed by Escherichia coli has taste-modifying activity and glycosylation is not essential for this activity. Although there is no direct contribution to the taste-modifying activity by N-glycan, the hyper-mannosyl-type bulky $\mathrm{N}$-glycan attached in yeast may cause steric hindrance of the 
interaction between rMCL and the sweet taste receptor, hT1R2/hT1R3. Alisdair et al. [24] reported a similar case in which high-mannose-type N-glycans of the carbohydrate-binding module of Cellulomonas fimi xylanase blocked the binding of cellulose.

\section{3. The N-glycan-mediated blocking mechanism of the taste-modifying activity}

The N-glycan of nMCL is attached to Asn42 and Asn186 [3]. To analyze sugar-chain modification of yeast-expressed rMCL, we compared three mutated proteins, N42Q, N186Q, and N42-186Q (C47-92S), using gel shift analysis (Fig. 5). The effect of the N42-186Q mutation was analyzed in combination with C47-92S, which is a more highly-secretable mutation (paper in preparation), because the N42-186Q mutant was not secreted. $\mathrm{N}$-glycosylation is important for the biological function, folding and (or) secretion of some eukaryotic proteins $[25,26]$. In the case of MCL, N-glycosylation may be an effective factor in secretion [6].

The band of the N42Q mutant was narrow at $50 \mathrm{kDa}$ and the molecular size slightly increased to reach that of the N42-186Q (C47-92S) non-glycosylated mutant (44 kDa). In contrast, the major band of the N186Q mutant was broad at $180 \mathrm{kDa}$ and the molecular size was similar to that of the wild-type. These results indicate that the sugar-chain structure at the position of Asn186 is relatively small and nearly homogenous, while that at Asn42 is large and shows micro-heterogeneity. The glycosylation sites of yeast-expressed rMCL were Asn42 and Asn186 only. In nMCL, 7 to 13 sugar units are attached per glycosylation site [3]. While the glycosylation sites of rMCL are identical to those of $\mathrm{nMCL}$, the volumes of the sugar chains were substantially different from those of nMCL. This suggests the possibility that the bulkiness of the N-glycan attached to Asn42 blocks the taste-modifying activity of rMCL.

Dimerization of rMCL is necessary for its taste-modifying activity [6,7]. His30, a candidate active residue [6], was located at the interface of the two monomeric MCL subunits in our recently published simulation model (Fig. 6) [6]. In this model, Asn42 was also located at the interface of the two monomeric MCLs. The bulky N-glycan attached to this glycosylation site may cause steric hindrance of the interaction between the interface active residues and the sweet taste receptor. The expression level of the N42Q mutant was poor and could not as yet be purified. The aims of our future investigations include both 3-D structure determination and mutagenetic analysis of the subunit interface residues. 


\section{Acknowledgements}

This study was supported in part by the ERATO Iwata Human Receptor Crystallography Project (JST) (to S.I.), the Targeted Proteins Research Program (to S.I. and T.M.), a Grant-in-Aid for Scientific Research (B) (20370035) (to T.K.), a grant from the Research and Development Program for New Bio-Industry Initiatives (to K.A.), Grants-in-Aid for Scientific Research (S) (to K.A.), Grants-in-Aid for JSPS Fellows (to A.K.) and Grants-in-Aid for JSPS Fellows (to K.I.) in Japan.

\section{References}

[1] S. Theerasilp, Y. Kurihara, Complete purification and characterization of the taste-modifying protein, miraculin, from miracle fruit, J. Biol. Chem. 263 (1988) 11536-11539.

[2] S. Theerasilp, H. Hitotsuya, S. Nakajo, K. Nakaya, Y. Nakamura, Y. Kurihara, Complete amino acid sequence and structure characterization of the taste-modifying protein, miraculin, $\mathrm{J}$. Biol. Chem. 264 (1989) 6655-6659.

[3] N. Takahashi, H. Hitotsuya, H. Hanzawa, Y. Arata, Y. Kurihara, Structural study of asparagine-linked oligosaccharide moiety of taste-modifying protein, miraculin, J. Biol. Chem. 265 (1990) 7793-7798.

[4] H. Igeta, Y. Tamura, K. Nakaya, Y. Nakamura, Y. Kurihara, Determination of disulfide array and subunit structure of taste-modifying protein, miraculin, Biochim. Biophys. Acta 1079 (1991) 303-307.

[5] K. Kurihara, L. M. Beidler, Mechanism of the Action of Taste-modifying Protein. Nature 222 (1969) 1176-1179.

[6] K. Ito, T. Asakura, Y. Morita, K. Nakajima, A. Koizumi, A. Shimizu-Ibuka, K. Masuda, M. Ishiguro, T. Terada, J. Maruyama, K. Kitamoto, T. Misaka, K. Abe, Microbial production of sensory-active miraculin, Biochem. Biophys. Res. Commun. 360 (2007) 407-411.

[7] T. Matsuyama, M. Satoh, R. Nakata, T. Aoyama, H. Inoue, Functional expression of miraculin, a taste-modifying protein in Escherichia coli, J. Biochem. 145 (2009) 445-450.

[8] H. J. Sun, M. L. Cui, B. Ma, H. Ezura, Functional expression of the taste-modifying protein, miraculin, in transgenic lettuce, FEBS Lett. 580 (2006) 620-626.

[9] S. Newstead, H. Kim, G. von Heijne, S. Iwata, D. Drew, High-throughput fluorescent-based optimization of eukaryotic membrane protein overexpression and purification in Saccharomyces cerevisiae, Proc. Natl. Acad. Sci. USA 104 (2007) 13936-13941. 
[10] T. Sugawara, K. Ito, M. Shiroishi, N. Tokuda, H. Asada, T. Yurugi-Kobayashi, T. Shimamura, T. Misaka, N. Nomura, T. Murata, K. Abe, S. Iwata, T. Kobayashi, Fluorescence-based optimization of human bitter taste receptor expression in Saccharomyces cerevisiae, Biochem. Biophys. Res. Commun. 382 (2009) 704-710.

[11] K. Ito, T. Sugawara, M. Shiroishi, N. Tokuda, A. Kurokawa, T. Misaka, H. Makyio, T. Yurugi-Kobayashi, T. Shimamura, N. Nomura, T. Murata, K. Abe, S. Iwata, T. Kobayashi, Advanced method for high-throughput expression of mutated eukaryotic membrane proteins in Saccharomyces cerevisiae, Biochem. Biophys. Res. Commun. 371 (2008) 841-845.

[12] A. L. Demain, P. Vaishnav, Production of recombinant proteins by microbes and higher organisms, Biotechnol. Adv. 27 (2009) 297-306.

[13] Y. Masuda, S. Nirasawa, K. Nakaya, Y. Kurihara, Cloning and sequencing of a cDNA encoding a taste-modifying protein, miraculin, Gene 161 (1995) 175-177.

[14] A. Grote, K. Hiller, M. Scheer, R. Münch, B. Nörtemann, D. C. Hempel, D. Jahn, JCat: a novel tool to adapt codon usage of a target gene to its potential expression host, Nucleic Acids Res. 33 (2005) W526-531.

[15] J. Kota, C. F. Gilstring, P. O. Ljungdahl, Membrane chaperone Shr3 assists in folding amino acid permeases preventing precocious ERAD, J. Cell Biol. 176 (2007) 617-628.

[16] T. Sahara, T. Gouda, Y. Tochigi, S. Ohgiya, A highly efficient secretory signal peptide and a protein expression system using the peptide thereof, European Patent (2007) EP1790661.

[17] W. K. Huh, J. V. Falvo, L. C. Gerke, A. S. Carroll, R. W. Howson, J. S. Weissman, E. K. O'Shea, Global analysis of protein localization in budding yeast, Nature 425 (2003) 686-691.

[18] F. Grueninger-Leitch, A. D'Arcy, B. D'Arcy, C. Chène, Deglycosylation of proteins for crystallization using recombinant fusion protein glycosidases, Protein Sci. 5 (1996) 2617-2622.

[19] E. Quartley, A. Alexandrov, M. Mikucki, F. S. Buckner, W. G. Hol, G. T. DeTitta, E. M. Phizicky, E. J. Grayhack, Heterologous expression of L. major proteins in S. cerevisiae: a test of solubility, purity, and gene recoding, J. Struct. Funct. Genomics 10 (2009) 233-247.

[20] M. Welch, S. Govindarajan, J. E. Ness, A. Villalobos, A. Gurney, J. Minshull, C. Gustafsson, Design parameters to control synthetic gene expression in Escherichia coli, PLoS One 4 (2009) e7002.

[21] P. M. Sharp, E. Cowe, D. G. Higgins, D. C. Shields, K. H. Wolfe, F. Wright, Codon usage patterns in Escherichia coli, Bacillus subtilis, Saccharomyces cerevisiae, Schizosaccharomyces pombe, Drosophila melanogaster and Homo sapiens; a review of the considerable within-species diversity, Nucleic Acids Res. 16 (1988) 8207-8211. 
[22] Y. Hagihara, P. S. Kim, Toward development of a screen to identify randomly encoded, foldable sequences, Proc. Natl. Acad. Sci. USA 99 (2002) 6619-6624.

[23] A. Herscovics, P. Orlean, Glycoprotein biosynthesis in yeast, FASEB J. 7 (1993) 7540-7550.

[24] A. B. Boraston, R. A. Warren, D. G. Kilburn, Glycosylation by Pichia pastoris decreases the affinity of a family 2 a carbohydrate-binding module from Cellulomonas fimi: a functional and mutational analysis, Biochem J. 58 (2001) 423-430.

[25] C. Reichling, W. Meyerhof, M. Behrens, Functions of human bitter taste receptors depend on N-glycosylation, J. Neurochem. 106 (2008) 1138-1148.

[26] Y. Liu, A. Nguyen, R. L. Wolfert, S. Zhuo, Enhancing the secretion of recombinant proteins by engineering N-glycosylation sites, Biotechnol. Prog. 25 (2009) 1468-1475. 


\section{Figure legends}

Fig. 1. Illustration of the signal-sequence optimization system. The mixture of $\mathbf{1 2}$ signal-sequence fragments, and target (MCL) gene fragment, were co-transformed into yeast. These fragments were then linked at a specific gene region encoding a $3 \times$ glycine and FLAG-tag (GGG DYKDDDDK) by homologous recombination. More highly-secretable signal-sequences were screened at the next step of on-plate immunodetection using anti-FLAG antibody.

Fig. 2. Codon optimization of the gene encoding MCL. a) Before, and b) after optimization. c) Secretion of native-type and codon-optimized MCL using the yeast expression system. Culture supernatant was probed by immunoblotting analysis using anti-MCL antibody [6]. The broad band at approximately $180 \mathrm{kDa}$ was glycosylated rMCL. d) Improvement of expression level and codon adaptation index (CAI) [14]. Open column, CAI; filled columns, expression level. The expression levels are shown as means \pm standard deviation $(n=4)$.

Fig. 3. Optimization of secretion signal-sequence. a) On-plate immunodetection of signal-sequence screening constructs. Colonies of transformants (left), the results of on-plate immunodetection using anti-FLAG antibody (middle), and the merged image (right) are shown. b) Screening of more highly-secretable signal-sequence. Twelve strongly-positive clones were selected from the plate and the adapted signal-sequences of these clones were confirmed by sequencing. To evaluate the expression level from each signal-sequence in liquid conditions, culture supernatant was probed by immunoblotting analysis using anti-MCL antibody [6]. The expression levels are shown as means \pm standard deviation $(n=4)$.

Fig. 4. Deglycosylation of purified MCL. a) SDS-PAGE analysis. Purified nMCL, rMCL and dgMCL were analyzed by SDS-PAGE with Coomassie Brilliant Blue (CBB) staining in the absence or presence of dithiothreitol (DTT). Black and gray arrowheads show dimeric- and monomeric-MCL, respectively. b) Recovery of the taste-modifying activity by deglycosylation. 
See Materials and Methods section for information on how the sweetness scores were defined. Sweetness scores were represented according to the concentration of aspartame: score 9, >2 $\mathrm{mM}$; score 8, $2 \mathrm{mM}$; score 7, 1-2 mM; score 6, $1 \mathrm{mM}$; score 5, 0.5-1 mM; score 4, $0.5 \mathrm{mM}$; score 3, 0.25-0.5 mM; score 2, $0.25 \mathrm{mM}$; and score $1,<0.25 \mathrm{mM}$. Black, gray and white columns show $\mathrm{pH} 3.0, \mathrm{pH} 5.0$ and $\mathrm{pH}$ 7.0, respectively. The sweetness scores are shown as means \pm standard deviation $(n=3)$.

Fig. 5. Mutation analysis at two glycosylation sites, Asn42 and Asn186. Culture supernatant was probed by immunoblotting analysis using anti-MCL antibody [6].

Fig. 6. Simulation model of MCL. The simulation model of MCL was as described previously [6]. The representative model is shown as a ribbon model. The glycosylation sites (Asn42 and Asn186), candidate active residue (His30) and cysteine residues forming the disulfide bond (Cys47-Cys92, Cys138-Cys138, Cys148-Cys159 and Cys152-155,) are shown by a stick model. Asparagine residues, magenda; histidine residue, yellow; cysteine residues, black. 


\section{Fig. 1}

Signal sequences

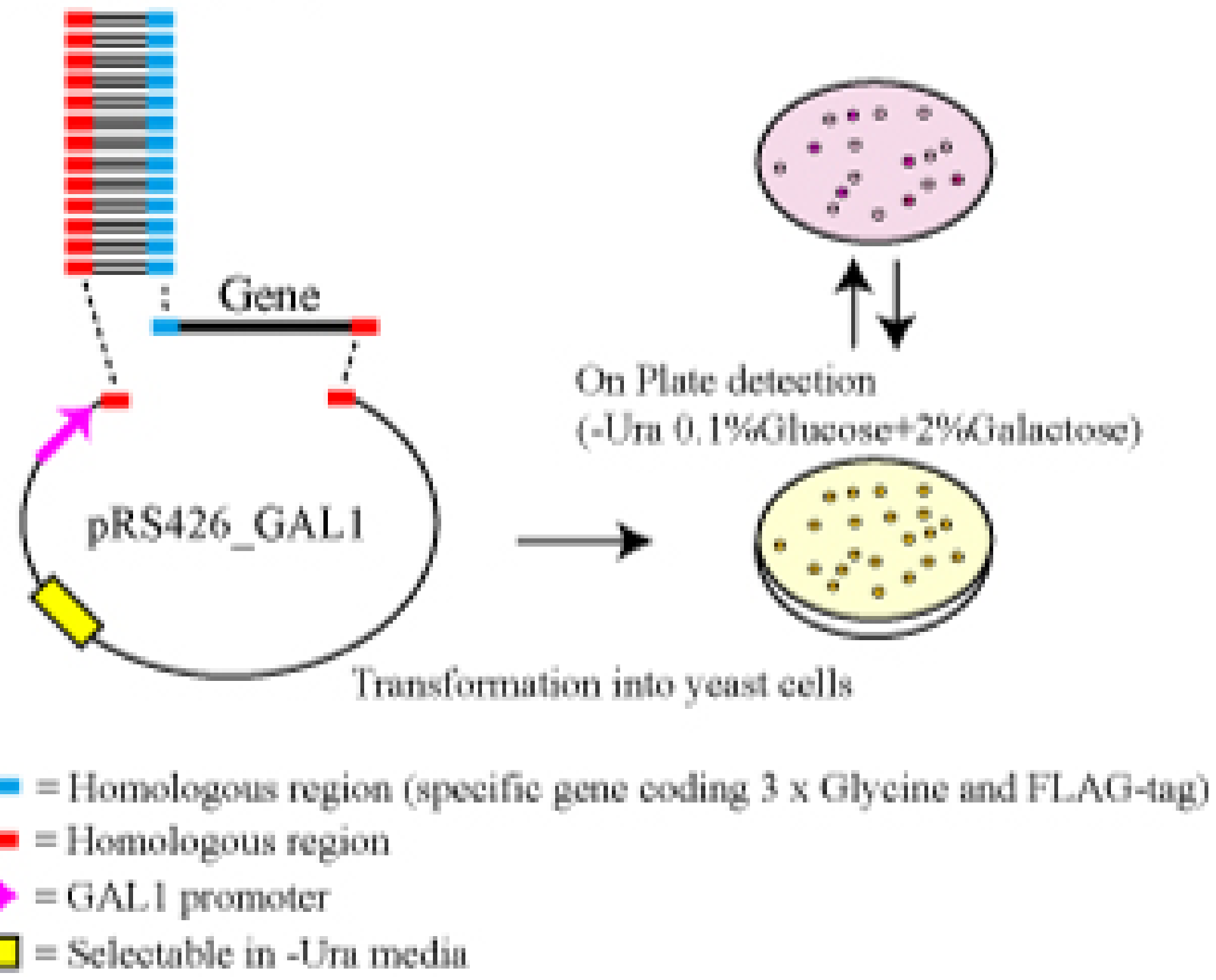




\section{Fig. 2}

a)

GAT TCG GCA CCC AAT COZ GTT CTT GAC ATA GAC QGA GAG AAA CTC COG ACG GOZ ACC AAT TAT TAC ATT GTO $D O B$ GTI CTC COS GAC CAT GQC OAC GOC CTT ACA GTA TCC GCC ACC ACC CCC AAC GOC ACC TTC GTT TOT CCA CEC ACA ITT GTC CAA ACA COA AAG GAO GTE GAC CAC GAT OQC COC CTC QCT TTE TTT CCA GAO AAC CCA AAO GAA CAC OTT GTT COA GTE TCC NCE GAT CTC NAC ATC MAT TTE TCO GCO TTC ATO CCO TGT CGT TGO ACC MOT TCE AOC GTO TOG CGO OTC GAC AAA TAC GAT GAA TOC ACG GOS CAG TAC TTC GTC ACC ATC OAC GGT GTC AAA GCA AAC CCA GOT OCC GAA ACC ATT AGT ACC TCC TTT AAG ATT GAG CAG TTT TCT CGT AGT GGT TTT TAC AAC CTT GTT TTC TGT CCC ACC

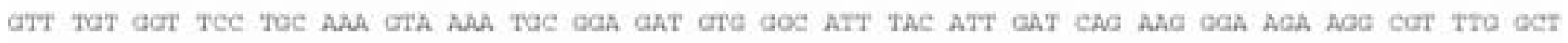
CTC AGC GAT MA CCA TTC GCA TTC GAO TTC AAC AM ACC GTA TAC TTC

b)

GAT TCT GCT CCA NA CCA GTT TTS GAC ATT GAC QOT GM MAA TTO MGA ACT GOT ACT MAT TAT TAC ATT GTT CCA GTT TTC AGA GAC CAT OGT OKA GGT TTO ACT GTT TCT GCT ACT ACT CCA AAC GCT ACT TTC GTT TOT CCA CCT AGA

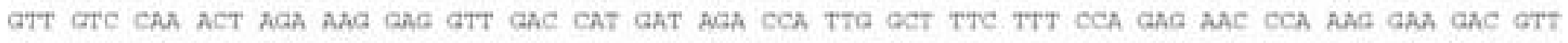

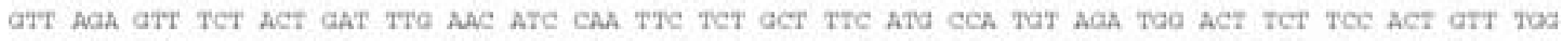
AOA TTO GAC AMA TAC GAT GNA TCT ACT QOT CAN TAC TTE GTT ACT ATE GaT GOT GTT NA GOT ANC CCA GaT COT GAA ACT ATT TCT TOC TOG TTT AAG ATT GAG GAG TTT TGT GST TCT GOT TTT TAC AAG TTC GTT TTC TCT CCA ACT GIT TOT GGT TCT TCT AAA GTT AAA TUT GCT CAT CTT GGT ATT TAC ATT CAT CAA AAC COT ACA AGA ACA TTC DCT TTO TCT GAT AAA CCA TTE OET TTE GAC TTE AAL AAA ACT GTT TAC TTE

c)

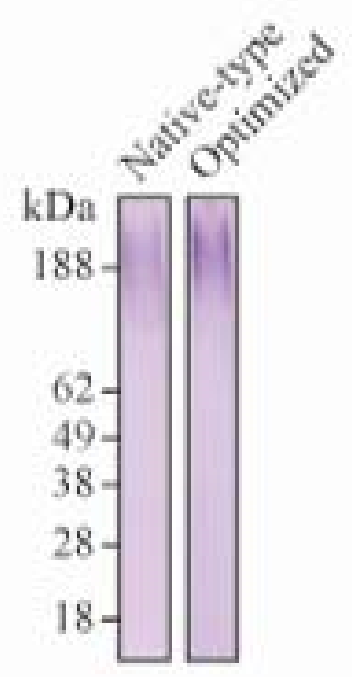

d)

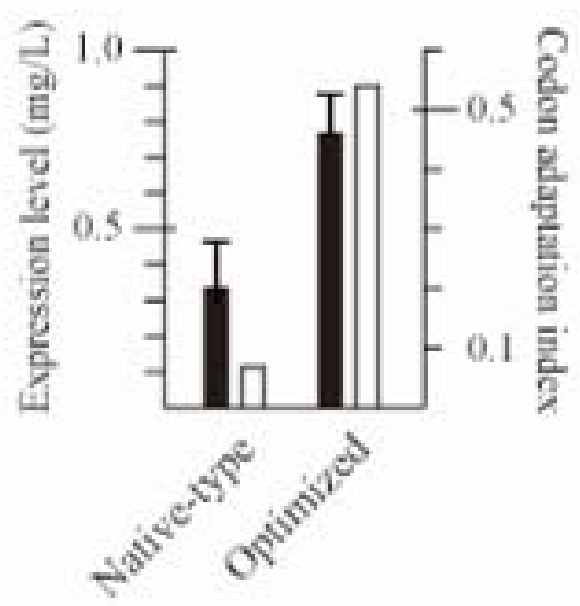


Fig. 3

a)
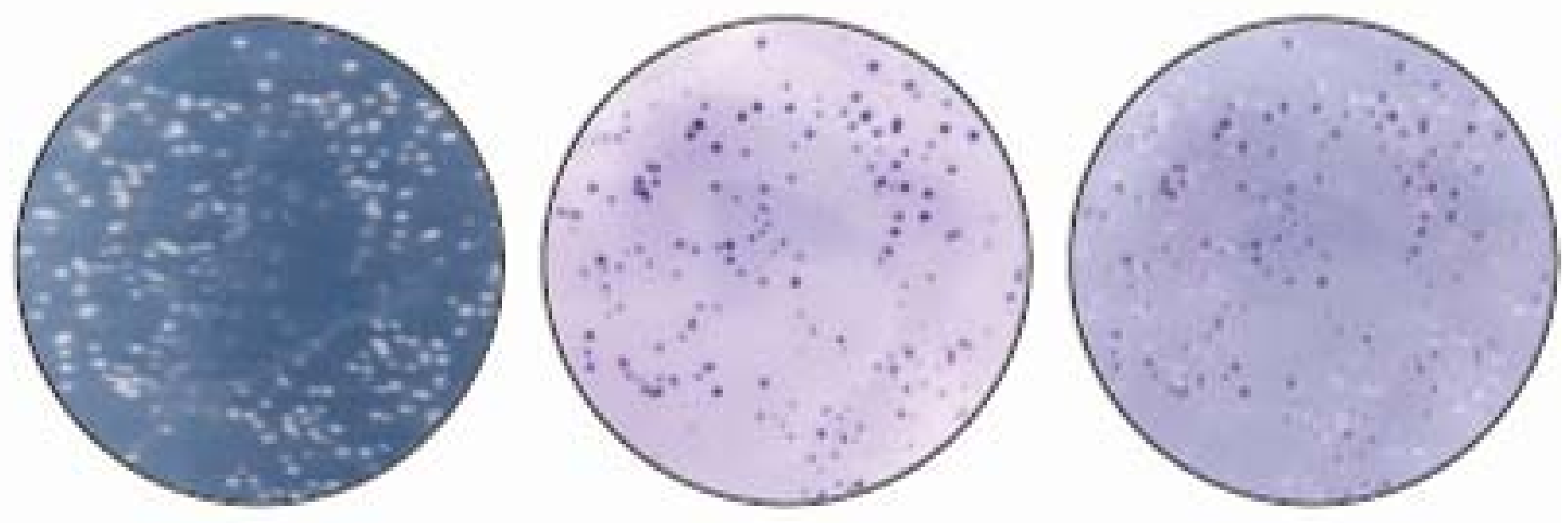

b)

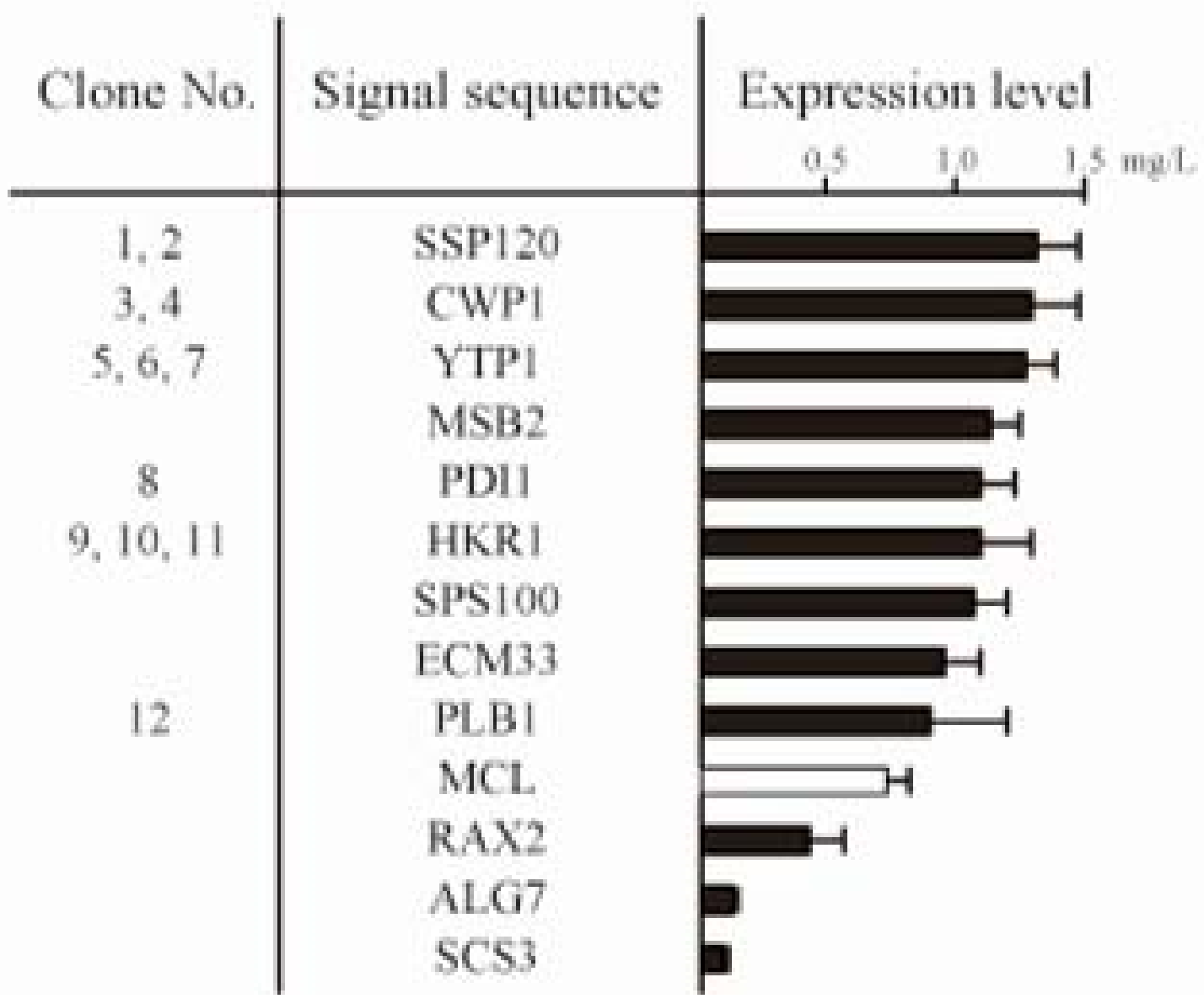


Fig. 4

11)

IMCL IMCL dg-rMCL

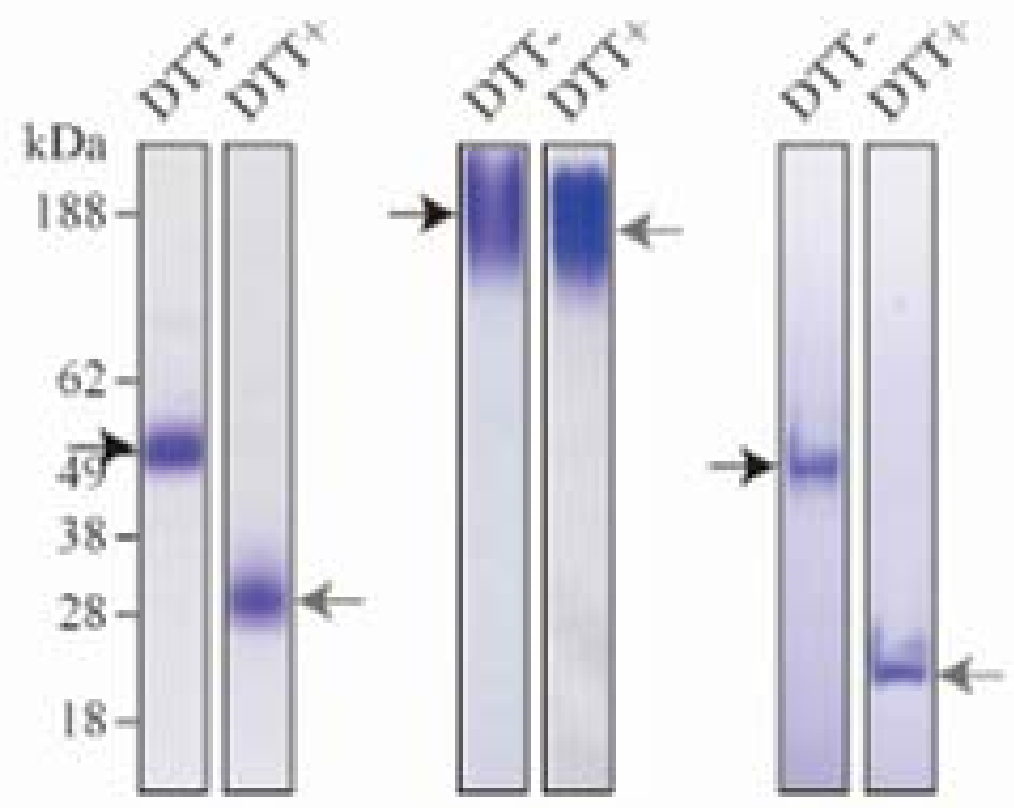

b)

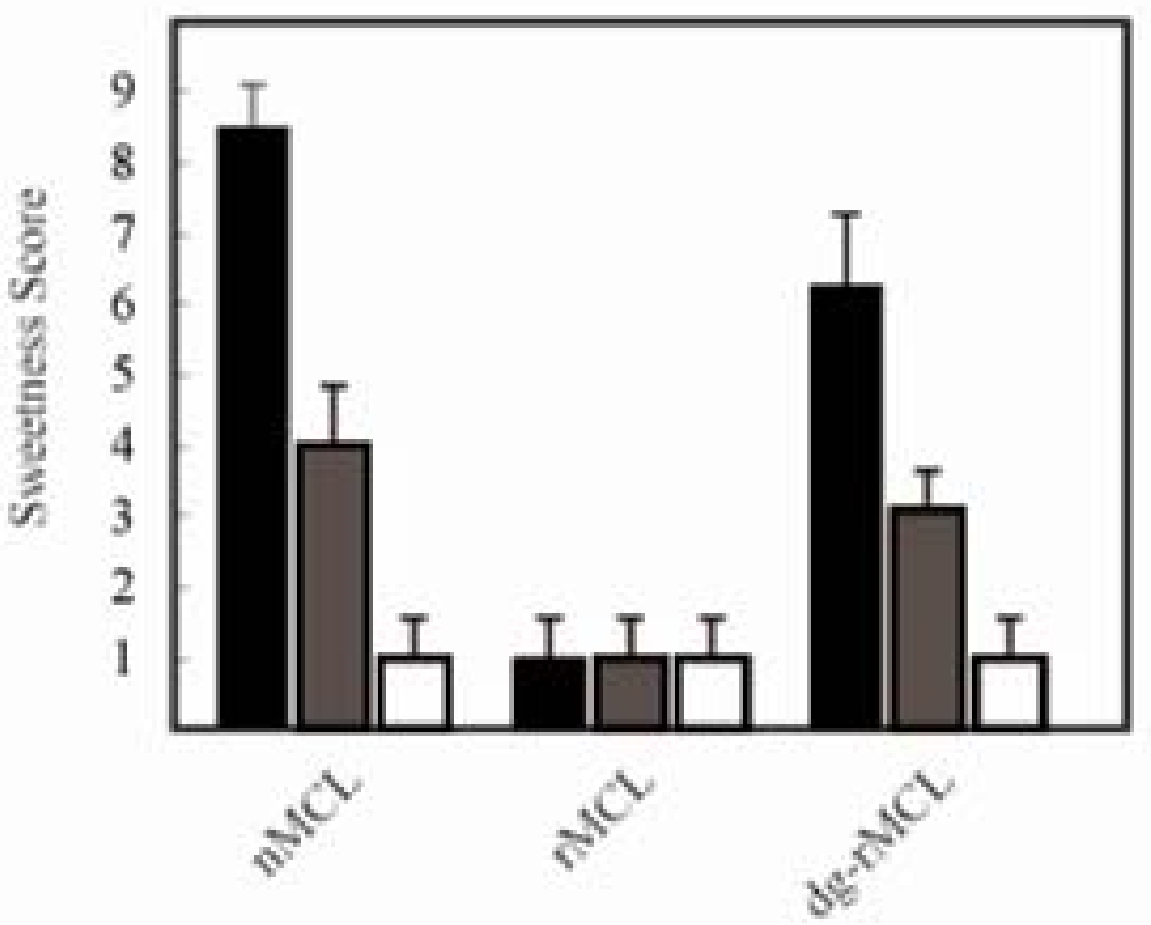




\section{Fig. 5}

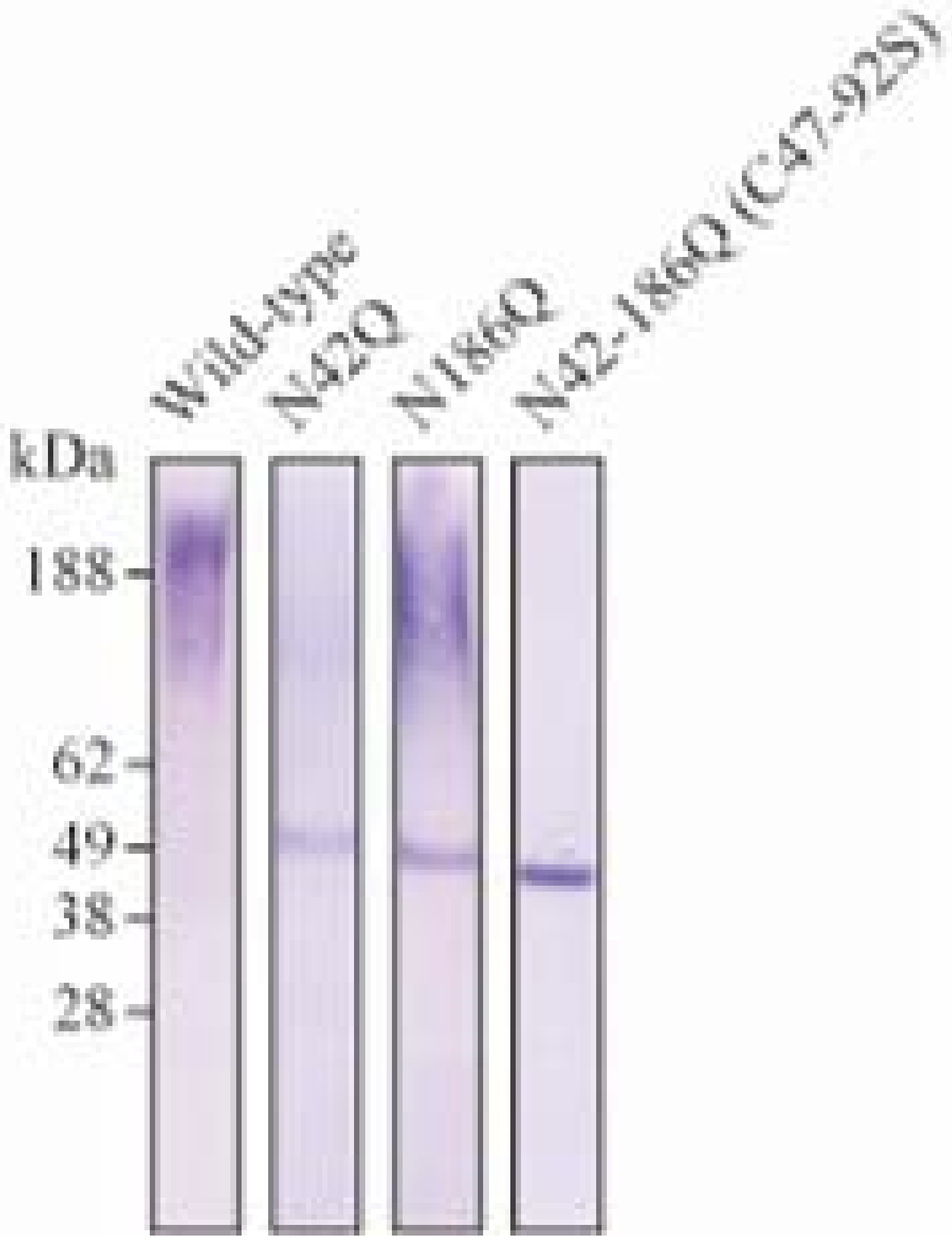


Fig. 6

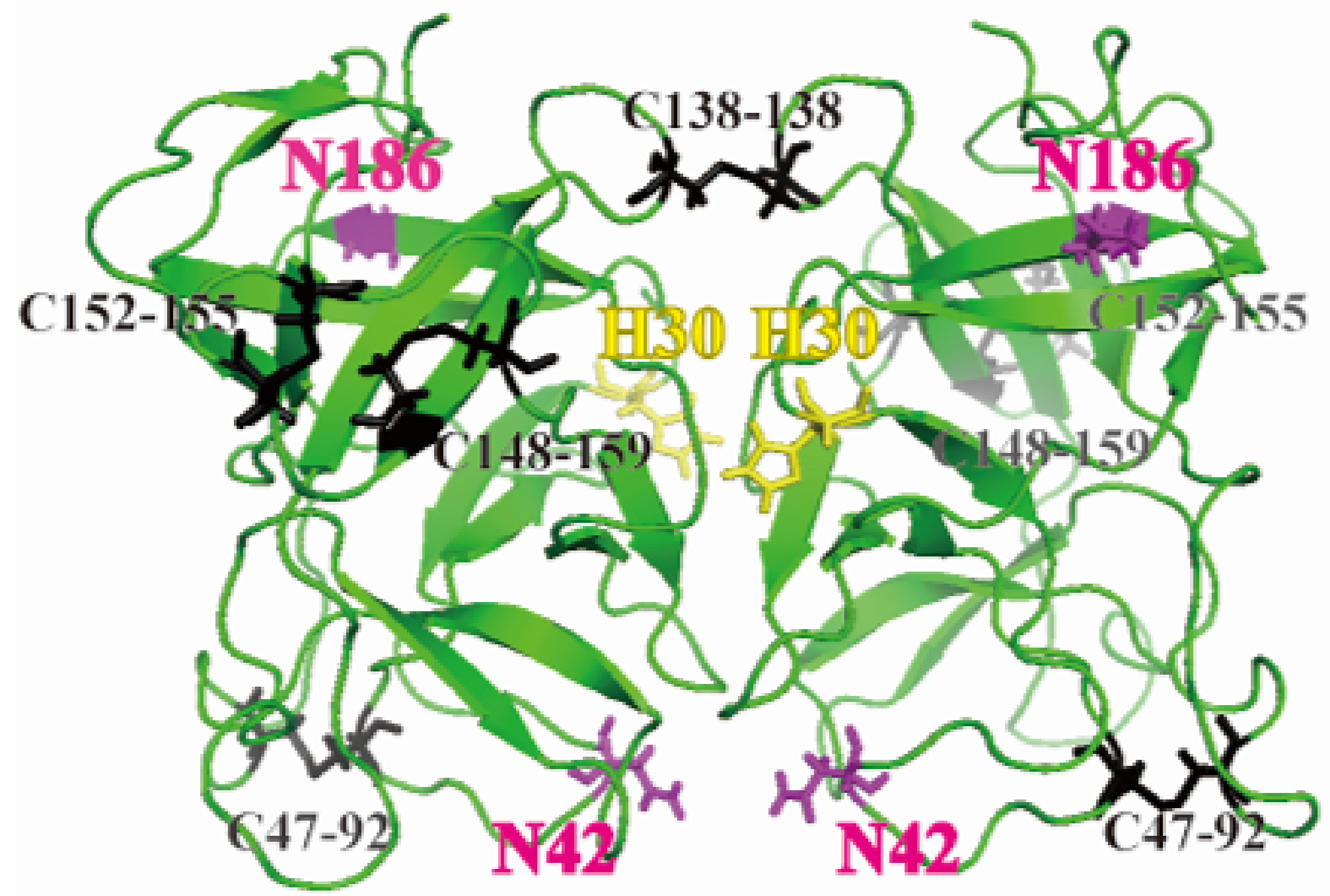




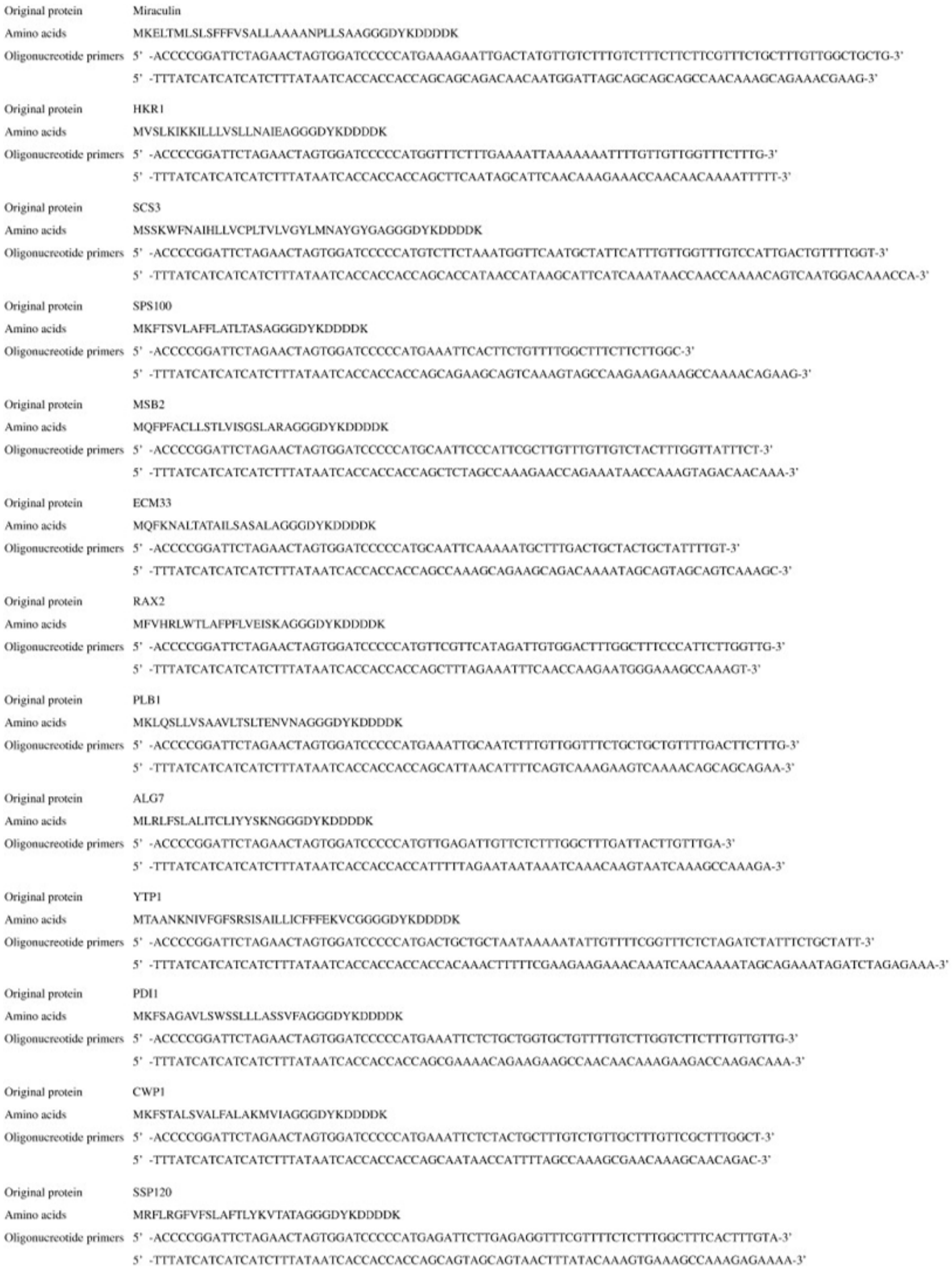

